\title{
Internal evaluation of risk stratification tool using procalcitonin and clinical risk factors in pediatric febrile neutropenia: the first step in a pilot quality improvement project
}

\author{
Charles Nessle ${ }^{1}$, Thomas Braun ${ }^{2}$, Sung Won Choi ${ }^{3}$, and Rajen Mody ${ }^{4}$ \\ ${ }^{1}$ University of Michigan Medical School \\ ${ }^{2}$ University of Michigan School of Public Health \\ ${ }^{3}$ University of Michigan Michigan Medicine \\ ${ }^{4}$ University of Michigan
}

October 20, 2021

\begin{abstract}
Risk stratification of pediatric febrile neutropenia (FN) is an established concept; the internal evaluation of a validated clinical decision rules (CDR) tool has not been well-described. In this study, restrictive criteria and procalcitonin were added to a recommended CDR for internal evaluation before implementation. Analysis of $577 \mathrm{FN}$ episodes showed good sensitivity and negative predictive value in predicting blood stream infections $(87.3 \% ; 95.6 \%)$ and intensive care admissions $(97.2 \%$; 99.1\%). There were no severe adverse events in low-risk patients with low procalcitonin; procalcitonin identified 3 low-risk patients with serious bacterial infections. The modified CDR with procalcitonin may assist in risk stratification.

"Internal evaluation of risk stratification tool using procalcitonin and clinical risk factors in pediatric febrile neutropenia: the first step in a pilot quality improvement project"

CN Nessle DO ${ }^{1}$, T Braun $\mathrm{PhD}^{2}$, SW Choi MD MS${ }^{1}, \mathrm{R}$ Mody MD MS ${ }^{1}$

${ }^{1}$ University of Michigan, Division of Pediatric Hematology Oncology, Department of Pediatrics

${ }^{2}$ University of Michigan, School of Public Health, Department of Biostatistics

Corresponding author:

C. Nathan Nessle DO
\end{abstract}

MPB D 4202

1500 E. Medical Center Dr., SPC 5718

Ann Arbor, MI 48109

Phone: (734) 647-2893

Fax: (734) 615-0464

Email: cnessle@med.umich.edu

Word count, abstract: 100

Word count, main text: 1200 
Number of tables and figures: 2

Number of supporting information files: 2

Short running title: Internal evaluation of FN risk stratification tool

Key words: febrile neutropenia, bloodstream infection, risk stratification

Abbreviations Table

\begin{tabular}{ll}
\hline FN & Febrile Neutropenia \\
COG & Children's Oncology Group \\
CDR & Clinical Decision rules \\
ANC & Absolute neutrophil count \\
BSI & Blood stream infection \\
IRB & Institutional Review Board \\
CVL & Central venous line \\
PICU & Pediatric intensive care unit \\
\hline
\end{tabular}

Abstract:

Risk stratification of pediatric febrile neutropenia (FN) is an established concept; the internal evaluation of a validated clinical decision rules (CDR) tool has not been well-described. In this study, restrictive criteria and procalcitonin were added to a recommended CDR for internal evaluation before implementation. Analysis of $577 \mathrm{FN}$ episodes showed good sensitivity and negative predictive value in predicting blood stream infections $(87.3 \% ; 95.6 \%)$ and intensive care admissions $(97.2 \% ; 99.1 \%)$. There were no severe adverse events in low-risk patients with low procalcitonin; procalcitonin identified 3 low-risk patients with serious bacterial infections. The modified CDR with procalcitonin may assist in risk stratification.

Introduction:

Febrile neutropenia (FN) is a frequent complication of myelosuppressive chemotherapy in pediatric cancer patients associated with blood stream infections and high mortality. ${ }^{1,2}$ Heterogeneity between FN episodes was observed leading to the novel concept of risk stratification and descriptions of clinical factors associated with severe outcomes. ${ }^{3}$ Patients at low-risk for severe outcomes can safely receive de-escalated treatment with oral antibiotics at home in either a step-down or upfront outpatient manner. ${ }^{4-8}$ Guidelines from the Children's Oncology Group (COG), Infectious Disease Society of America, and National Cancer Center Network recommend adoption and clinical incorporation of a validated clinical decision rules (CDR) risk stratification tool to aid in the identification of low-risk patients. ${ }^{9-11}$ A CDR validated in a certain population and region may not have a similar performance in a different region and population, attributed to variations in supportive care and chemotherapy regimens, differences in local antibiogram, and subjective criterion required in some tools. ${ }^{10,12,13}$

Given the difficulty in validating a CDR, serum inflammatory biomarkers allow for objective data which may help in identifying high-risk patients. ${ }^{14} \mathrm{~A}$ review of over 8300 pediatric FN episodes concluded that procalcitonin $<0.5 \mathrm{ng} / \mathrm{mL}$ was the best performing serum biomarker early in the infection course; serial trending may further improve accuracy in screening for adverse events. ${ }^{14-16}$ Improved performance of CDR and success rates in step-down therapy are observed when biomarkers are combined with a clinical risk factors. ${ }^{16-18}$

The primary aim of this study was to describe our experience of the internal evaluation of a CDR; an important, "required" step recommended by COG prior to implementation. ${ }^{10}$

Methods:

Design and Definitions 
This cohort study utilized a composite retrospective and prospective design. We used our hospital electronic medical record (EPIC, MiChart) to generate a report of FN admissions for a 34-month window, then prospective non-intervention observation for a 10-month period. Included episodes met the standard definition of FN in the presence of a central venous line (CVL). ${ }^{9}$ Episodes were excluded if they did not meet FN definition or those which occurred near stem cell engraftment to restrict the analytic group to the general oncology cohort. Severe adverse events were defined as diagnosis of bloodstream infection (BSI), pediatric intensive care unit (PICU) admission, or infection related death.

\section{Risk Stratification}

Risk stratification was assigned based on initial presentation using a modified version of a CDR validated in similar populations and recommended by COG. ${ }^{10,18-20}$ Expanded high-risk clinical factors were added to reflect our center's local population and acuity similar to prior studies. ${ }^{21}$ Procalcitonin with a threshold of $<2 \mathrm{ng} / \mathrm{mL}$ at presentation or $<0.4 \mathrm{ng} / \mathrm{mL}$ after one night as was incorporated to aid in BSI screening. ${ }^{16}$ (See Table 1 )

\section{Statistical Analysis}

Continuous demographics were summarized with medians and ranges, while categorical demographics were summarized with proportions. Differences between the retrospective FN and prospective FN groups were determined with a Wilcoxon Rank Sum test and chi-squared test of association for continuous and categorical characteristics, respectively. AUC values were computed from a logistic regression model. All analyses were done in the statistical software R, version 4.0.4, and statistical significance was defined as a $p$-value less than 0.05 .

\section{Ethical and Regulatory Oversight}

This study was reviewed and deemed exempt by the University of Michigan Institutional Review Board (IRB). Data and private health information were stored on IRB-approved secure hard-drives.

Results

\section{Performance of the modified risk tool without procalcitonin}

The performance of the modified CDR using only the clinical risk criterion was analyzed in 577 FN episodes (Table 2) . There were $460 \mathrm{FN}$ episodes analyzed in the retrospective group and $117 \mathrm{FN}$ episodes analyzed prospectively; $80 \%(462 / 577)$ presented with outpatient fevers. The modified CDR identified $60.5 \%$ $(349 / 577)$ as high-risk episodes encompassing most severe events; $19.8 \%(69 / 349)$ had a BSI and $19.8 \%$ required PICU admission (69/349). In 228 low-risk patients, $4.4 \%(10 / 228)$ were diagnosed with a BSI (sensitivity $=87.3 \%$; $\mathrm{NPV}=95.6 \%$; $\mathrm{AUC}=0.63)$ and $0.9 \%(2 / 228)$ required PICU admission (sensitivity $=$ $97.2 \% ; \mathrm{NPV}=99.1 \% ; \mathrm{AUC}=0.70)$.

In the prospective group, there were significantly fewer low-risk $(30.8 \%[36 / 117]$ vs. $41.7 \%[192 / 460] ; P=$ $0.03)$ and more high-risk episodes $(69.2 \%[81 / 117]$ vs. $58.3 \%[268 / 460] ; P=0.03)$. No differences were detected in BSI $(15.4 \%$ [18/117] vs. $13.3 \%$ [61/460]; $P=0.55)$ or PICU admissions $(13.7 \%[16 / 117]$ vs. $12 \%$ $[55 / 460] ; P=0.61)$. Significantly fewer upper respiratory infections were diagnosed $(9.4 \%[11 / 117]$ vs. $18.9 \%$ $[87 / 460] ; P=0.01)$. No significant differences were observed in the demographics, malignancy classification, or type of CVL (Supplemental Table 1).

Prospective Analysis of the complete modified risk tool with procalcitonin

Analysis of the modified CDR with serial procalcitonin of $<2 \mathrm{ng} / \mathrm{mL}$ or $<0.4 \mathrm{ng} / \mathrm{mL}$ was performed in $88 \%$ (103/117) of patients; median time from fever onset to the first and second procalcitonin values was 2 and 16 hours, respectively. There were no serious adverse events in low-risk episodes with low procalcitonin at either time point; AUC for BSI and PICU was 0.79 and 0.83 . The CDR with procalcitonin stratified $79.2 \%$ $(78 / 96)$ and $77 \%(77 / 100)$ as high-risk at either time point; 23 patients were eligible for early discharge. There were 2 low-risk episodes diagnosed with BSI fromStreptococcus mitis ; procalcitonin was elevated at 
both time points $(0.26 \mathrm{ng} / \mathrm{mL}$ and $4.32 \mathrm{ng} / \mathrm{mL} ; 0.35 \mathrm{ng} / \mathrm{mL}$ and $0.71 \mathrm{ng} / \mathrm{mL})$. A third low-risk episode with elevated procalcitonin $(21.2 \mathrm{ng} / \mathrm{mL}$ and $20.74 \mathrm{ng} / \mathrm{mL})$ was readmitted in 5 days due to a soft tissue abscess fromActinomyces requiring surgical intervention. Two high-risk episodes with BSI from methicillin Staphylococcus aureus andRothia mucilaginosa had low procalcitonin values $(0.06 \mathrm{ng} / \mathrm{mL}$ and $0.08 \mathrm{ng} / \mathrm{mL}$; $0.18 \mathrm{ng} / \mathrm{mL}$ and $0.5 \mathrm{ng} / \mathrm{mL}$ ).

Discussion:

Febrile neutropenia guidelines recommend implementation of a validated CDR to identify low-risk patients, and this report describes the important stage of tool selection and internal validation necessary prior to clinical implementation. The clinical criteria of the modified CDR identified 39.5\% of patients as low-risk FN with a similar severe adverse event rate in low-risk patients as described in prior studies. ${ }^{19,21}$ It is not clear if we recorded fewer low-risk episodes in the prospective observation due to fluctuations in patient acuity, significantly less viral infections, or study design.

There were no severe adverse events in low-risk patients with low procalcitonin. Procalcitonin correctly identified BSI in two low-risk patients and a surgical abscess in another. The AUC analysis of the various models showed the CDR improved with procalcitonin, but few serious outcomes limited further conclusions. The implementation of risk stratification in FN patients represents a significant change in practice for our center; the additional procalcitonin data decreased patients eligible for early discharge but shows promise in detecting BSI in low-risk patients. We improved sensitivity of the CDR to decrease misclassification of a low-risk patient for upfront outpatient management or early inpatient discharge at implementation. Our group will perform ongoing evaluation of the CDR during implementation as a quality improvement project with the intentions of augmenting the discharge criteria based on the safety analysis.

The modified CDR performed well with improved discretion with addition of procalcitonin. This internal evaluation was not only an imperative step in determining the tool's clinical discretion in our local patient population, but also a highly regarded necessary evaluation in changing our clinical practice. Internal evaluation of a CDR is an important step recommended by COG to evaluate both performance in the local population and to provide foundation for safe implementation when changing clinical practice.

Conflicts of Interest: The authors report no relevant conflicts of interest.

Acknowledgements:

C.N.N. acknowledges support from a NIH training grant (T32CA236621-01A1).

Contribution statement:

All authors equally contributed to the study. CNN conceptualized the study. CNN drafted the manuscript, performed literature review and data collection, assisted in interpretation of data, and assisted in editing of report. TB performed data analysis and assisted with interpretation of data. SWC and RM assisted with interpretation of data, editing of report, and oversaw conduct of the study.

References:

1. Bodey GP, Buckley M, Sathe YS, Freireich EJ. Quantitative relationships between circulating leukocytes and infection in patients with acute leukemia. Ann Intern Med. 1966;64(2):328-340.

2. Mueller EL, Croop J, Carroll AE. Fever and neutropenia hospital discharges in children with cancer: A 2012 update. Pediatr Hematol Oncol. 2016;33(1):39-48.

3. Talcott JA, Finberg R, Mayer RJ, Goldman L. The medical course of cancer patients with fever and neutropenia. Clinical identification of a low-risk subgroup at presentation. Arch Intern Med.1988;148(12):25612568.

4. Morgan JE, Cleminson J, Atkin K, Stewart LA, Phillips RS. Systematic review of reduced therapy regimens for children with low risk febrile neutropenia. Support Care Cancer. 2016;24(6):2651-2660. 
5. Orme LM, Babl FE, Barnes C, Barnett P, Donath S, Ashley DM. Outpatient versus inpatient IV antibiotic management for pediatric oncology patients with low risk febrile neutropenia: a randomised trial.Pediatr Blood Cancer. 2014;61(8):1427-1433.

6. Robinson PD, Lehrnbecher T, Phillips R, Dupuis LL, Sung L. Strategies for Empiric Management of Pediatric Fever and Neutropenia in Patients With Cancer and Hematopoietic Stem-Cell Transplantation Recipients: A Systematic Review of Randomized Trials. J Clin Oncol.2016;34(17):2054-2060.

7. Stern A, Carrara E, Bitterman R, Yahav D, Leibovici L, Paul M. Early discontinuation of antibiotics for febrile neutropenia versus continuation until neutropenia resolution in people with cancer. Cochrane Database Syst Rev. 2019;1:CD012184.

8. Vidal L, Ben Dor I, Paul M, et al. Oral versus intravenous antibiotic treatment for febrile neutropenia in cancer patients. Cochrane Database Syst Rev. 2013(10):CD003992.

9. Baden LR, Swaminathan S, Angarone M, Blouin G. NCCN Clincal Practice Guidelines in Oncology: Prevention and Treatment of Cancer Related Infections. 2016; https://oralcancerfoundation.org/wpcontent/uploads/2016/09/infections.pdf.

10. Lehrnbecher T, Robinson P, Fisher B, et al. Guideline for the Management of Fever and Neutropenia in Children With Cancer and Hematopoietic Stem-Cell Transplantation Recipients: 2017 Update. J Clin Oncol. 2017;35(18):2082-2094.

11. Taplitz RA, Kennedy EB, Flowers CR. Outpatient Management of Fever and Neutropenia in Adults Treated for Malignancy: American Society of Clinical Oncology and Infectious Diseases Society of America Clinical Practice Guideline Update Summary. J Oncol Pract.2018;14(4):250-255.

12. Haeusler GM, Thursky KA, Slavin MA, et al. Risk stratification in children with cancer and febrile neutropenia: A national, prospective, multicentre validation of nine clinical decision rules.EClinicalMedicine. 2020;18:100220.

13. Phillips B, Morgan JE, Haeusler GM, Riley RD, Collaborative P. Individual participant data validation of the PICNICC prediction model for febrile neutropenia. Arch Dis Child. 2020;105(5):439-445.

14. Arif T, Phillips RS. Updated systematic review and meta-analysis of the predictive value of serum biomarkers in the assessment and management of fever during neutropenia in children with cancer.Pediatr Blood Cancer. 2019;66(10):e27887.

15. Mian A, Becton D, Saylors R, et al. Biomarkers for risk stratification of febrile neutropenia among children with malignancy: a pilot study. Pediatr Blood Cancer. 2012;59(2):238-245.

16. Reitman AJ, Pisk RM, Gates JV, 3rd, Ozeran JD. Serial procalcitonin levels to detect bacteremia in febrile neutropenia. Clin Pediatr (Phila). 2012;51(12):1175-1183.

17. Miedema KG, Tissing WJ, Abbink FC, et al. Risk-adapted approach for fever and neutropenia in paediatric cancer patients-a national multicentre study. Eur J Cancer. 2016;53:16-24.

18. Santolaya ME, Alvarez AM, Aviles CL, et al. Early hospital discharge followed by outpatient management versus continued hospitalization of children with cancer, fever, and neutropenia at low risk for invasive bacterial infection. J Clin Oncol. 2004;22(18):3784-3789.

19. Alexander SW, Wade KC, Hibberd PL, Parsons SK. Evaluation of risk prediction criteria for episodes of febrile neutropenia in children with cancer. J Pediatr Hematol Oncol. 2002;24(1):38-42.

20. Santolaya ME, Alvarez AM, Acuna M, et al. Efficacy and safety of withholding antimicrobial treatment in children with cancer, fever and neutropenia, with a demonstrated viral respiratory infection: a randomized clinical trial. Clin Microbiol Infect.2017;23(3):173-178. 
21. Paolino J, Mariani J, Lucas A, et al. Outcomes of a clinical pathway for primary outpatient management of pediatric patients with low-risk febrile neutropenia. Pediatr Blood Cancer. 2019;66(7):e27679.

TABLE 1 The modified risk tool recommended by COG with additional restrictive clinical criteria specific to our local patient population and acuity. Procalcitonin was added as objective biomarker inflammatory data as an additional restrictive high-risk criterion to aid in diagnosis of bacteremia.

TABLE 2 Hematopoietic cell transplant $(\mathrm{HCT})=$ a patient encounter on the Blood and marrow transplant (BMT) service. MDI (microbiologically documented infection) encompassed blood stream infections, upper respiratory infections, C. difficile colitis, fungal infection, and urinary tract infections as well as clinically documented infections. Short length-of-stay (SLOS) was defined as a hospital stay [?]3 days. LOS = lengthof-stay. PICU = pediatric intensive care unit.

SUPPLEMENTAL TABLE 1 The calculated $p$-value compared differences of the FN episodes in the retrospective group with FN episodes in the prospective group. Location describes setting of first fever event. $\mathrm{AML}=$ acute myelogenous leukemia. $\mathrm{ALL}=$ acute lymphoblastic leukemia. $\mathrm{CVL}=$ central venous line. PICC $=$ peripherally inserted central catheter

\section{Hosted file}

TABLE 1.docx available at https://authorea.com/users/442111/articles/542452-internalevaluation-of-risk-stratification-tool-using-procalcitonin-and-clinical-risk-factorsin-pediatric-febrile-neutropenia-the-first-step-in-a-pilot-quality-improvement-project

\section{Hosted file}

TABLE 2.docx available at https://authorea.com/users/442111/articles/542452-internalevaluation-of-risk-stratification-tool-using-procalcitonin-and-clinical-risk-factorsin-pediatric-febrile-neutropenia-the-first-step-in-a-pilot-quality-improvement-project 